\title{
Research Progress on Antibiosis Activity of Scutellaria Flavonoids
}

\author{
Jun Yuan Qin ${ }^{1}$, Wen Xiu Liu', Xiao Fang Xie ${ }^{1 \star}$ and Peng Cheng ${ }^{1 \star}$
}

School of Pharmacy, Chengdu University of Traditional Chinese Medicine, 37 Shierqiao Road, Jinniu District, 611137 Chengdu, China

\section{Asian Journal of Complementary and Alternative Medicine. Volume 09 Issue 4}

Published on: $04 / 11 / 2021$

*Author for Correspondence: Peng Cheng, School of Pharmacy, Chengdu University of Traditional Chinese Medicine, 37 Shierqiao Road, Jinniu District, 611137 Chengdu, China, E-mail: pengchengchengdu@126.com

Xiao Fang Xie, School of Pharmacy, Chengdu University of Traditional Chinese Medicine, 37 Shierqiao Road, Jinniu District, 611137 Chengdu, China, E-mail: xiexiaofang@cdutcm.edu.cn

Cite this article as: Qin JY, Liu WX, Xie XF, Cheng P. Research Progress on Antibiosis Activity of Scutellaria Flavonoids. Asian Journal of Complementary and Alternative Medicine, Vol 9(4), 100-105:2021.

\section{ABSTRACT}

Scutellariae (Huangqin) is a traditional medicine that is widely applied in China for the treatment of cold and fever, and nowadays research found that it can against pathogenic microorganism. Flavonoids is the main active constituent isolated from Scutellariae and possesses outstanding antibiosis activity. Scutellaria flavonoids displayed antiseptic actions and anti-resistance, including but not limited to destroy bacterial biofilm, suppressed activity of the DNA enzyme, restrain efflux pump and quorum-sensing system. This paper highlights and discusses the antibiosis activity of Scutellaria flavonoids, along with possible mechanisms of its action. hoping to providing basis and evidence for clinical application.

Keywords: Scutellaria; Scutellaria flavonoids; Bacteria; Antibiosis

\section{INTRODUCTION}

Scutellariae (Huangqin), the dried root of Scutellaria baicalensis Georgi, is a widespread used herb in clinic to treat cold, fever, and infectious diseases in china [1]. In the past decades, researchers found that scutellariae display various bioactivity including anti-inflammatory, antiviral, neuroprotection and antibiosis [2]. Flavonoids are class of compounds with rich pharmacological activities and have excellent antibacterial performance,which is main active constituent of scutellariae [3]. Nowadays, baicalin has been regarded as one of the index components in scutellariae, and its content should not be less than $8.0 \%$ percent in scutellariae according to Pharmacopoeia of the People's Republic of China [1]. Modern research has found baicalin can inhibit the growth of Staphylococcus aureus, Escherichia coli, Pseudomonas aeruginosa, and Candidicoccus albicans, which via destroying the bacterial biofilm, reduce the expression of bacterial virulence factors. Not only baicalin but also baicalein and wogonoside have excellent antibacterial effects, and their chemical structure is shown in Figure 1. Herein, the antibiosis of Scutellariae flavonoids were discussed, and even more the mechanism。<smiles>O=C(O)[C@H]1O[C@@H](Oc2cc3oc(-c4ccccc4)cc(=O)c3c(O)c2O)[C@H](O)[C@@H](O)[C@@H]1O</smiles>

(a)<smiles>COc1c(O[C@@H]2O[C@H](C(=O)O)[C@@H]([18OH])[C@H](O)[C@H]2O)cc(O)c2c(=O)cc(-c3ccccc3)oc12</smiles>

(b)<smiles>O=c1cc(-c2ccccc2)oc2cc(O)c(O)c(O)c12</smiles>

(c)

Figure 1: The chemical structures of Baicalin (a), Wogonoside (b) and Baicalein (c). 


\section{Staphylococcus aureus}

Staphylococcus aureus (SA) is a conditionally pathogenic gram-positive bacterium, which causing infectious diseases such as pericarditis, cephalomeningitis and septicemia [4]. The minimum inhibitory concentration(MIC) of baicalin and baicalein were $1 \mathrm{mg} / \mathrm{mL}$ and $0.25 \mathrm{mg} / \mathrm{mL}$ [5]. Baicalin can increase the conductivity of and nucleic acid leakage of SA in the concentration of $1 \mathrm{~g} / \mathrm{mL}$, at the same time, thallus were withered and broken [6]. Baicalin can suppressed the formation of biofilm and damage the cell membrane of SA [7]. Baicalin can inhibit the expression of many virulence factors of SA. Wang et al.found that baicalin were interaction with residues $\mathrm{Asn}^{92}$ and $\mathrm{Tyr}^{128}$ which directly binds to the active center of the key virulence factor, Sortase B (SrtB) [8]. Moreover, baicalin impeded the cell lysis activity of $\alpha$-hemolysin (Hla) by way of combining with the Y148, P151, and F153 of Hla [9]. In animals, it was enhanced bacterial clearance and survival rate via attenuating the expression of inflammatory factors, increasing the number of lymphocytes and dendritic cells and inhibiting the apoptosis of tissue cells [10-12].

Interestingly, baicalein also showed significant inhibition on methicillin-resistant Staphylococcus aureus (MRSA), the MIC were $0.64 \mu \mathrm{g} / \mathrm{mL}$ [13]. Further studies showed, it embeds and binds DNA to inhibit the activity of DNA isomeric topoisomerase, resulting in DNA replication and transcription abnormalities and loss of biological function in vivo, but does not affect the integrity of MRSA cells and cell walls $[14,15]$. Reversal of bacterial resistance to $\beta$-lactam antibiotics against MRSA at a baicalein dose of $5 \mathrm{mg} / \mathrm{mL}$ [16]. This effect caused by restraining NorA efflux pump, suppressing MRSA-specific pyruvate kinase lead to deficiency of ATP and attenuating SOS-response [17,18]. Baicalein integrates with Asp-75 and Lys-80 residues binding to Von Willebrand factor-binding protein $(\mathrm{vWbp})$ which affected bacteria proliferation [19]. chen et al. found that baicalein treatment reduced staphylococcal enterotoxin A (SEA) and Hla levels, downregulated the quorum-sensing system regulators agrA, RNAIII, and sarA, and gene expression of ica [20]. Baicalein inhibited the accessory gene regulator system, reducing the expression of SEA, thus lowering CRP and PCT levels [21].

\section{Pseudomonas aeruginosa}

Pseudomonas aeruginosa (PA), is a conditionally pathogenic Gram-negative bacterium, which induced infectious disease including endocardial infection, urinary tract infection, gangrene pustulosis [22]. Baicalin inhibited the growth of PA biofilm and reduced bacterial adhesion at $0.625 \mathrm{mg} / \mathrm{mL}$; baicalin $(3.75 \mathrm{mg} / \mathrm{ml})$ diminished the expression of LasR, RhlR and PvdQ in quorum-sensing (QS)systems system, which is closely related to biofilm formation of PA. baicalin $(15.65 \mathrm{mg}$ / $\mathrm{mL}$ ) can destroy biofilm of Pseudomonas aeruginosa; When the concentration reaches $32.25 \mathrm{mg} / \mathrm{L}$, baicalin had significant effects in interfering with the biofilm formation of PA, reducing the yield of polysaccharide-protein complexes, and inhibiting the expression of (QS)Las1m RNAm [23-26]. In addition, baicalin attenuated the expression of 3-oxo-C12-HSL and C4-HSL of QS signaling [27]. Moreover, baicalin suppressed the functional genes of PA such as virulence genes,quorum sensing genes and motility related genes [28].

Baicalein inhibited the formation of bacterial biofilm and suppressed the adhesion of PA at a concentration of $2 \mu \mathrm{g} / \mathrm{mL}$ [29]. When the concentration were increased to $64 \mu \mathrm{g} / \mathrm{mL}$, it suppressed that clust movement and twitching movement ability of PA [30,31]. Baicalein reduces the secretion of inflammatory factors in macrophages by inhibiting the activation of PA-infected macrophage MAPK and NFkB signal transduction pathways [32].

\section{Escherichia coli}

Escherichia coli (E. coli), is a gram-negative bacterium which is an important strain of human intestinal flora, but at the same time, it can cause diarrhea, abdominal pain, fever and other diseases [33]. The MIC of baicalin were $512 \mathrm{mg} / \mathrm{mL}$, it were hindered the formation of E. coli biofilm and significantly reduce the adhesion gene fliC [34]. Moreover, baicalin attenuated cytotoxicity of $\mathrm{E}$. coli via forming a pentamer with Shiga toxins 1 and 2 (Stx1 and Stx2) [35]. When the concentration were 4-40 mM, baicalein promoted hydrolysis of receptor TraR protein [36]. Baicalein can inhibit the activity of ATP synthase and the synthesis of ATP in E. coli [37].

\section{Helicobacter pylori}

Helicobacter pylori (Hp), a microaerobic Gram-negative bacteria, was first isolated from the gastric mucosa by Australian scholars Warren and Mashall in 1983. Hp is closely related to the occurrence of a variety of digestive diseases, which can lead to stomach cancer, gastric mucosarelated lymphoma, whereupon the World Health Organization therefore identified it as a Class I carcinogen in 1994 [38]. The MIC90 and MIC50 of jaundice for 10 clinical Hp strains were $1.31 \mathrm{mg} / \mathrm{mL}, 1.05 \mathrm{mg} / \mathrm{mL}$, and the MIC for multi-drug-resistant $\mathrm{Hp}$ strains was $25-200 \mathrm{mg} / \mathrm{mL}[39,40]$. Baicilin inhibits the pyroenose of $\mathrm{Hp}$, which binds to the flap region of the pyroenose, preventing the flap region from shutting down [41]. And through the thiohydro bond with the space with the key with the customs Cys321 located on the mobileflap [42]. High expression of hefA gene, one of the coding active exosotysis systems, is associated with HP's resistance, and baicilin can 
reduce the expression of the hefA gene [43]. Baicalin and baicalin inhibited the expression of $\mathrm{Hp}$ vacuolating cytotoxin A (vacA), a type of gastric epithelial cell that causes the gastric epithelial cells to bubble [44]. In Hp-induced human gastric epithelial GES-1 cells, baicalin $(60 \mathrm{mg} / \mathrm{L})$ reduces the inflammation factors secreted by GES-1 cells infected with HP, promotes cell proliferation and reduces apoptosis. Further studies demonstrated that this is associated with inhibition of p38 MAPK pathways [45].

\section{Acinetobacter baumannii}

Acinetobacter baumannii (AB), a Gram-negative bacterium, which is a strict oxygen demand, non-lactose fermentation condition pathogenic bacteria [46]. The MICs of baicalein and wogonoside were 0.625 and $0.125 \mathrm{mg} / \mathrm{mL}$, and baicalein restrained the biofilm of $\mathrm{AB}$ [47]. Further research observed that baicalein stimulated the expression of adeA, adeR, adeS genes of efflux pump, OXA-23,OXA-51 genes of $\beta$-lactamase, and CarO,OmpAv of membrane protein [48].

\section{Klebsiella pneumoniae}

Klebsiella pneumoniae (Kpn) has capsuleis, is a Gramnegativeand and opportunistic pathogen that is native to the gastrointestinal tract and the pharynx [49]. The MIC of baicalin were $6.250 \mathrm{mg} / \mathrm{mL}$, and it suppressed biofilm formation [50,51]. CTX-M is a dominant gene for antibiotic resistance to $\beta$-lactamase in broad-spectrum $\beta$-lactamase Kpn,the combination therapy of baicalein and cefotaxime can remarkably decrease transcribed mRNA level of CTX-M-1 [22].

\section{Salmonella typhimurium}

Salmonella typhimurium (S. Typhimurium) is a Gramnegative, spore-less anaerobic bacteria, which is closely related to diarrhea [52]. Baicalien diminished S. Typhimurium on epithelial cells by hindering S.Typhimurium pathogenicity island-1 (SPI-1) type III secretion system (T3SS) and translocase at 50M [53]. And baicalien attenuated the levels of tumor necrosis factor $\alpha(\mathrm{TNF}-\alpha)$, nitrate, and lactate dehydrogenase (LDH) from S. typhimurium-infected Caco-2 cells [54]. Moreover,baicalin and baicalein can significantly restrain the transcription levels of sopB, sopE, and sopE2 genes which are associated with the Salmonella pathogenicity island 1 virulence S. Typhimurium [54].

\section{Mycobacterium tuberculosis}

Mycobacterium tuberculosis (Mtb), is the main pathogen of tuberculosis [55]. Baicalin has a significant inhibitory effect on $\mathrm{Mtb}$, the MICs were $0.75 \sim 12 \mathrm{mg} / \mathrm{m}$ [56]. For clinically resistant bacteria, the MICs of baicalin for 37 clinically isolated strains was $1.5 \mathrm{~g} / \mathrm{L}$, with a maximum greater than $48 \mathrm{~g} / \mathrm{L}$ [57]. The concentration of baicalin increased the morphological change of Mycobacterium tuberculosis, and the degree of damage was increasing [58]. Further studies have found that baicalin is not only attached to the surface of Mtb, but also in the inside of Mtb to play a role, the site of action is cytoplasm [59]. In Mtb infected mice, baicalin activate inflammasomes and inducing autophagy of host macrophages [60].

\section{Candidicoccus albicans}

Candidicoccus albicans (C.albicans), a single-celled eukaryote, most exists in the body's gastrointestinal tract, mouth, and urethra [61]. The MIC of baicalin were $1.0 \mathrm{mg} /$ $\mathrm{mL}$, it inhibits the formation and adhesion of the C.albicans tube $[25,62]$. Baicalin affected the synthesis of DNA, RNA, and proteins by inhibiting leucine in bacteria [63]. Baicalin induced the nuclear solidification and fragmentation of C.albicans cell, and it lowed the expression of mitochondrial membrane potential(MMP), and enhanced the CAP1,SOD2 and TRR1 genes which are relevant to redox and the level of reactive oxygen species (ROS) [64,65]. Besides, baicalin affected the cell cycle and ultra-microstructure of candida biofilm cells, hindered cell growth cycles, and disrupted their mitochondrial function, even induced biofilm apoptosis via activating Cytochrome $\mathrm{C}(\mathrm{Cyt} \mathrm{C})$ and metacaspase $[66,67]$. The combination with fluconazole reduces hydrophobicity on the surface of the biofilm [68]. In addition, the MICs of baicalein ranged from 13 to $104 \mu \mathrm{g} / \mathrm{mL}$ of six C.albicans [69].

\section{Bacteria associated with periodontitis}

The MICs of baicalin were 1.0,2.0 and $2.0 \mathrm{~g} / \mathrm{L}$, and the MBCs were 2.0, 8.0 and $4.0 \mathrm{~g} / \mathrm{L}$ of Porphyromonas gingivalis (P.g), Fusobacterium nucleatum (F.n) and Aggregatibacter actinomycetemcomitans (A.a) [70]. When the concentration of baicalin reached $500 \mu \mathrm{g} / \mathrm{mL}$, it hindered formation biofilm of streptococcus mutans which is the main caries pathogen, and inhibited the production of acids [71]. In periodontitis rats, baicalin reduced inflammatory responses in rats with laboratory periodontitis and the alveolar bone loss via inhibition toll-like receptor 2(TLR2) and toll-like receptor 4 (TLR4) expression and downstream signaling [72].

\section{CONCLUSION}

In recent years, as an antibacterial and treatment of infectious diseases, Chinese medicine has received widespread attention because of its good efficacy and its difficulty in producing drug resistance. The growth of bacteria and the formation of biofilms are inhibited by flavonoids of scutellariae. Of note, flavonoids inhibited the activity of enzymes in DNA, affecting the metabolism of bacteria and the synthesis of 
macromolecular substances. More importantly, for resistant bacteria, flavonoids inhibited efflux pump and quorum sensing. In animals, flavonoids diminished adhesion to the host and increased the removal rate of host bacteria, also reduces the host's inflammatory response. This makes it promising to be a clinically innovative drug. However, more data from preclinical studies are needed,including pharmacokinetics and pharmacodynamics. As well as its modern pharmacological research is limited to in vitro and animal experiments, has not been widely used and clinical, which to a certain extent limits the drug value.

\section{FUNDING}

The work was supported by the regional Joint Fund of National Natural Science Foundation of China: Study on the geo-herbalism of Medicinal Materials from Sichuan Tract (U19A2010).

\section{REFERENCES}

1. Chinese Pharmacopoeia Commission (2020) Pharmacopoeia of the People's Republic of China. China Medical Science and Technology Press.

2. Song JW, Long JY, Xie L, Zhang LL, Xie QX, et al. (2020) Applications, phytochemistry, pharmacological effects, pharmacokinetics, toxicity of Georgi. and its probably potential therapeutic effects on COVID-19: A review. Chin Med 15: 102.

3. Rahman MM, Rahaman MS, Islam MR, Hossain ME, Mithi FM, et al. (2021) Multifunctional Therapeutic Potential of Phytocomplexes and Natural Extracts for Antimicrobial Properties. Antibiotics (Basel) 10: 1076.

4. Lakhundi S, Zhang K (2018) Methicillin-Resistant Staphylococcus aureus: Molecular Characterization, Evolution, and Epidemiology. Clin Microbiol Rev 31: e00020-18.

5. Li X, Fang B (2014) Effect of seven monomeric components in scutellaria baicalensis georgi on staphylococcus aureus and streptococcus pneumoniae Study on the in vitro bacteriostatic activity of bacteria. Journal of Tianjin Medical University 20: 399-401.

6. Cui W (2020) Study onantibacterial activity and mechanism of different extracts and related components of Scutellariae Radix. Jinzhou Medical University.

7. Zhang S, Hu B, Xu J, Ren Q, Wang Z, et al. (2020) Baicalin suppress growth and virulence-related factors of methicillinresistant Staphylococcus aureus in vitro and vivo. Microb Pathog 139: 103899.

8. Wang G, Gao Y, Wang H, Niu X, Wang J (2018) Baicalin Weakens Pathogenicity by Targeting Sortase B. Front Cell Infect Microbiol 8: 418.

9. Qiu J, Niu X, Dong J, Wang D, Wang J, et al. (2012) Baicalin protects mice from Staphylococcus aureus pneumonia via inhibition of the cytolytic activity of $\alpha$-hemolysin. J Infect Dis 206: 292-301.
10. Zhu J, Wang J, Sheng Y, Zou Y, Bo L, et al. (2015) Baicalin improves survival in a murine model of polymicrobial sepsis via suppressing inflammatory response and lymphocyte apoptosis. PLoS One 7: e35523.

11. Guo M, Zhang N, Li D, Liang D, Liu Z, et al. (2013) Baicalin plays an anti-inflammatory role through reducing nuclear factor$\kappa \mathrm{B}$ and $\mathrm{p} 38$ phosphorylation in S. aureus-induced mastitis. Int Immunopharmacol 16: 125-130.

12. Guo M, Cao Y, Wang T, Song X, Liu Z, et al. (2014) Baicalin inhibits Staphylococcus aureus-induced apoptosis by regulating TLR2 and TLR2-related apoptotic factors in the mouse mammary glands. Eur J Pharmacol 723: 481-488.

13. Liu IX, Durham DG, Richards RM (2000) Baicalin synergy with beta-lactam antibiotics against methicillin-resistant Staphylococcus aureus and other beta-lactam-resistant strains of S. aureus. J Pharm Pharmacol 52: 361-366.

14. Chen Y, Zhou T, Xie K, Yun B, Xie M (2013) Antibacterial Mechanism of Baicalein on Methicillin-resistant Staphylococcus aureus. Chinese Journal of Animal and Veterinary Sciences 44: 2000-2006.

15. Yun B (2013) Antibacterial activity and mechanism of Baicalein on MRSA. Liaoning Normal University.

16. Eumkeb G, Sakdarat S, Siriwong S (2010) Reversing $\beta$-lactam antibiotic resistance of Staphylococcus aureus with galangin from Alpinia officinarum Hance and synergism with ceftazidime. Phytomedicine 18: 40-45.

17. Chan BCL, Ip M, Lau CBS, Lui SL, Jolivalt C, et al. (2011) Synergistic effects of baicalein with ciprofloxacin against NorA over-expressed methicillin-resistant Staphylococcus aureus (MRSA) and inhibition of MRSA pyruvate kinase. J Ethnopharmacol 137: 767-773.

18. Peng Q, Zhou S, Yao F, Hou B, Huang Y, et al. (2011) Baicalein suppresses the SOS response system of Staphylococcus aureus induced by ciprofloxacin. Cell Physiol Biochem 28: 1045-1050.

19. Zhang H, Luan Y, Jing S, Wang Y, Gao Z, et al. (2020) Baicalein mediates protection against Staphylococcus aureus-induced pneumonia by inhibiting the coagulase activity of vWbp. Biochem Pharmacol 178: 114024.

20. Chen Y, Liu T, Wang K, Hou C, Cai S, et al. (2016) Baicalein Inhibits Staphylococcus aureus Biofilm Formation and the Quorum Sensing System In Vitro. PLoS One 11: e0153468.

21. Liu T, Luo J, Bi G, Du Z, Kong J, et al. (2020) Antibacterial synergy between linezolid and baicalein against methicillin-resistant Staphylococcus aureus biofilm in vivo. Microb Pathog 147: 104411.

22. Cai W, Fu Y, Zhang W, Chen X, Zhao J, et al. (2016) Synergistic effects of baicalein with cefotaxime against Klebsiella pneumoniae through inhibiting CTX-M-1 gene expression. BMC Microbiol 16: 181.

23. Wang G, Fan Y, Wang L, Wu J (2011) Effect of Baicalin on pseudomonas aeruginosa Biofilms. Journal of Medical Research 40: 131-143.

24. Guo X (2020) Experimental study on the regulation effect of baicalin on the QS system of pseudomonas aeruginosa. Henan University of Chinese Medicine. 
25. Xie L, Zhou M, Chen Y, Xiong L, Xia P (2010) Study on Inhibition Effect of Baicalin and Baicalein on the Formation of Pseudomonas Aeruginosa Biofilm. China Pharmacy 21: 3651-3653.

26. Li S, Fang H, Chen Y, Gong S, Wang P, et al. (2017) Experimental study on intervention of baicalin and astragaloside IV on Pseudomonas aeruginosa biofilm in vitro. Shanghai Journal of Traditional Chinese Medicine 51: 63-83.

27. Luo J, Dong B, Wang K, Cai S, Liu T, et al. (2017) Baicalin inhibits biofilm formation, attenuates the quorum sensingcontrolled virulence and enhances Pseudomonas aeruginosa clearance in a mouse peritoneal implant infection model. PLoS One 12: e0176883.

28. Zhang P, Guo Q, Wei Z, Yang Q, Guo Z, et al. (2021) Baicalin Represses Type Three Secretion System of through PQS System. Molecules 26: 1497.

29. Li Y (2017) The Effect of Baicalein on Pseudomonas Aeruginosa Early Biofilm Infection in the Intraperitonel Cavity of Mouse. Guangxi Medical University.

30. Dong B, Kong J, Chen Y, Wang K, Luo J, et al. (2016) In vitro investigate of the inhibition of baicalein on Pseudomonas aeruginosa motility. Chinese Journal of Nosocomiology 26: 3361-3367.

31. Dong B (2016) The Intervention Effect of Baicalein and Baicalein of Biofilm of Pseudomnasaeruginosa In Vitro. Guangxi Medical University.

32. Luo J, Kong JL, Dong BY, Huang H, Wang K, et al. (2016) Baicalein attenuates the quorum sensing-controlled virulence factors of Pseudomonas aeruginosa and relieves the inflammatory response in P. aeruginosa-infected macrophages by downregulating the MAPK and NFкB signal-transduction pathways. Drug Des Devel Ther 10: 183-203.

33. Pakbin B, Brück WM, Rossen JWA (2021) Virulence Factors of Enteric Pathogenic: A Review. Int J Mol Sci 22: 9922.

34. Xie G, Duan Q, Xiang H, Hao J, Li W, et al. (2020) Effect of baicalin on expression of biofilm protein in escherichia coli. Lishizhen Medicine and Materia Medica Research 31: 1858-1862.

35. Vinh PT, Shinohara Y, Yamada A, Duc HM, Nakayama M, et al. (2019) Baicalein Inhibits Stx 1 and 2 of EHE: Effects of Baicalein on the Cytotoxicity, Production, and Secretion of Shiga Toxins of Enterohaemorrhagic Escherichia coli. Toxins (Basel) 11: 505.

36. Zeng Z, Qian L, Cao L, Tan H, Huang Y, et al. (2008) Virtual screening for novel quorum sensing inhibitors to eradicate biofilm formation of Pseudomonas aeruginosa. Appl Microbiol Biotechnol 79: 119-126.

37. Chinnam N, Dadi PK, Sabri SA, Ahmad M, Kabir MA, et al. (2010) Dietary bioflavonoids inhibit Escherichia coli ATP synthase in a differential manner. Int J Biol Macromol 46: 478-486.

38. Tirado-Hurtado I, Carlos C, Lancho L, Alfaro A, Ponce R, et al. (2019) Helicobacter pylori: History and facts in Peru. Crit Rev Oncol Hematol 134: 22-30.

39. Zhao J (2017) Effect of scutellaria baicalensis georgi on antibacterial activity of helicobacter pylori in vitro. Chinese Journal of Modern Drug Application 11: 195-196.
40. Huang Y, Huang G, Li X, Huang X, Wei L, et al. (2013) Effect on the Biofilm Formation of Drug. Resistant Helicobacter Pylori by Chinese Herbal Extracts Herald of Medicine 32: 1407-1409.

41. Yu X (2015) Biological Evaluation and Molecular Docking of Baicalin and Scutrllarin as Helicobacterpylori Urease Inhibitors. Guangzhou University of Chinese Medicine.

42. Yu XD, Zheng RB, Xie JH, Su JY, Huang XQ, et al. (2015) Biological evaluation and molecular docking of baicalin and scutellarin as Helicobacter pylori urease inhibitors. J Ethnopharmacol 162: 69-78.

43. Huang YQ, Huang GR, Wu MH, Tang HY, Huang ZS, et al. (2015) Inhibitory effects of emodin, baicalin, schizandrin and berberine on hefA gene: Treatment of Helicobacter pylori-induced multidrug resistance. World J Gastroenterol 21: 4225-4231.

44. Chen ME, Su CH, Yang JS, Lu CC, Hou YC, et al. (2018) Baicalin, Baicalein, and Lactobacillus Rhamnosus JB3 Alleviated Helicobacter pylori Infections in Vitro and in Vivo. J Food Sci 83: 3118-3125.

45. Deng Z, Wang Q (2017) Protective Effect and Mechanism of Baicalin in Alleviating Helicobacter Pylori-induced Human Gastric Epithelial GES-1 Cells Injury. Chinese Journal of Experimental Traditional Medical Formulae 23: 145-149.

46. da Fonseca ADS, Mencalha AL, de Paoli F (2021) Antimicrobial photodynamic therapy against Acinetobacter baumannii. Photodiagnosis Photodyn Ther 35: 102430.

47. Shen H, Zhang C, Lin H, Chen X, Huang R, et al. (2019)Antibacterial effect of flavonoids on resistant Acinetobacter baumannii. Natural Product Research and Developmen 31: 747-752.

48. Zhen Y (2021) Study on the mechanism of antibacterial effect in vitro of carbapenem-resistant Acinetobacter baumannii by the combination of baicalein and meropenem. Zunyi Medical University.

49. Opoku-Temeng C, Malachowa N, Kobayashi SD, Leo FRD (2021) Innate Host Defense against Klebsiella pneumoniae and the Outlook for Development of Immunotherapies. J Innate Immun 2021: 1-15.

50. Ou Y, Yu G, Fu Y (2015) Stuctural modification and bacteriostasis research of 8-(N,N-dimethyl-amine methyl )-baicalin. Chinese Journal of Pharmaceutical Analysis 35: 938-944.

51. We S, Wu J (2013) Effect of baicalein on growth and biofilm formation of klebsiella pneumoniae. Laboratory Medicine and Clinic 10: 2707-2708.

52. Patra SD, Mohakud NK, Panda RK, Sahu BR, Suar M (2021) Prevalence and multidrug resistance in Salmonella enterica Typhimurium: An overview in South East Asia. World J Microbiol Biotechnol 37: 185.

53. Tsou LK, Lara-Tejero M, Figura JR, Zhang ZJ, et al. (2016) Antibacterial Flavonoids from Medicinal Plants Covalently Inactivate Type III Protein Secretion Substrates. J Am Chem Soc 138: 2209-2218.

54. Wu SC, Chu XL, Su JQ, Cui ZQ, Zhang LY, et al. (2018) Baicalin protects mice against Salmonella typhimurium infection via the modulation of both bacterial virulence and host response. Phytomedicine 48: 21-31. 
55. Mekonnen D, Derbie A, Mihret A, Yimer SA, Tønjum T, et al. (2021) Lipid droplets and the transcriptome of Mycobacterium tuberculosis from direct sputa: A literature review. Lipids Health Dis 20: 129 .

56. Xiao H, Zhang X, Shao S, Zhang L, Zhang L (2017) Inhibitory effect of baicalin on mycobacterium tuberculosis. Chinese Journal of Clinical Laboratory Science 35: 291-292.

57. Shao S, Liu X, Sun W, Zhang L, Xiao H, et al. (2012) Study of Antituberculosis of Baicalin on Mycobacterium Tuberculosis. Tianjin Medical Journal 40: 763-765.

58. Zhou Q (2012) Research of mechanisman the antituberculosisc Action of baicalin. Tianjin Medical University.

59. Zhang X (2012) Research on the antituberculosis action of baicalin and mechanism. Tianjin Medical University.

60. Zhang Q, Sun J, Wang Y, He W, Wang L, et al. (2017) Antimycobacterial and Anti-inflammatory Mechanisms of Baicalin via Induced Autophagy in Macrophages Infected with. Front Microbiol 8: 2142.

61. Zolin GVS, Fonseca FHD, Zambom CR, Garrido SS (2021) Histatin 5 Metallopeptides and Their Potential against Candida albicans Pathogenicity and Drug Resistance. Biomolecules 11: 1209.

62. Wang C, Feng X, Zhang X, Zhu Q, Xi C, et al. (2010) Inhibitory effect of baicalin ongerm tube formation and alhesion of Candida albicans. China Journal of Chinese Materia Medica 35: 32163218.

63. Xiong Y, Fu Y, Kuang N, Zhang W (2004) Mechanism study of baicalin against Candida albicans. Chinese Pharmacological Bulletin 12: 1404-1407.
64. Huang S (2009) Mechanism study of baicalin against Candida albicans. Naval Medical University.

65. Feng X, Wang C, Wang T, Xu Z, Han N, et al. (2012) Apoptosis induced by baicalin inCandida albicans. Chinese Traditional Patent Medicine 34: 1443-1446.

66. Shi G (2015) Ras-Camp Pathway-Based Investigation of Inhibitory Effects and Mechanism of Baicalin Alone or in Combination with Fluconazole Against Candida Albicans Biofilms Ras-cAMP. Anhui University of Chinese Medicine.

67. Wang T, Shi G, Shao J, Wu D, Yan Y, et al. (2015) In vitro antifungal activity of baicalin against Candida albicans biofilms via apoptotic induction. Microb Pathog 87: 21-29.

68. Yan G, Shi G, Shao J, Wang T, Xia T, et al. (2015) Inhibitory effects of baicalin in cambination with fluconazole against Candida albicans biofilms. Chinese Journal of Mycology 10: 139-145.

69. Serpa R, França EJG, Furlaneto-Maia L, Andrade CGTJ, Diniz A, et al. (2012) In vitro antifungal activity of the flavonoid baicalein against Candida species. J Med Microbiol 61: 1704-1708.

70. Gong Y, Wang Z, Wu Z, Seng B, Wu Z, et al. (2021) Antibacterial effect of baicalin and its anti-inflammatory and osteogenic ability on experimental periodontitis in rats. Journal of Xinjiang Medical University 44: 415-420.

71. Elango AV, Vasudevan S, Shanmugam K, Solomon AP, Neelakantan P (2021) Exploring the anti-caries properties of baicalin against: An study. Biofouling 37: 267-275.

72. Sun JY, Li DL, Dong Y, Zhu CH, Liu J, et al. (2016) Baicalin inhibits toll-like receptor $2 / 4$ expression and downstream signaling in rat experimental periodontitis. Int Immunopharmacol 36: 86-93. 